\title{
Editorials
}

\section{DNase in cystic fibrosis: the challenge of assessing response and maximising benefit}

\author{
J Alastair Innes
}

Since nebulised recombinant human DNase was licensed for use in cystic fibrosis patients in March 1994, controversy has continued over how this relatively expensive drug should be used to maximum benefit. By cleaving DNA released from dead inflammatory cells it is dramatically effective in reducing sputum viscoelasticity in vitro. ${ }^{1}$ In vivo, phase 2 trials showed encouraging improvements in spirometric ${ }^{2}{ }^{3}$ and other inflammatory indices, ${ }^{4}$ and the major phase 3 trial used to support licensing ${ }^{5}$ showed that once daily treatment gave a relatively modest $(5.8 \%)$ improvement in forced expiratory volume in one second $\left(\mathrm{FEV}_{1}\right)$ at 24 weeks, with a significant (after age adjustment of the groups) reduction in exacerbations requiring parenteral antibiotics. This large randomised trial (968 patients) was notable, not only for the significance of the mean results, but also for the wide variability of individual responses to treatment. For example, $30 \%$ of actively treated patients showed an increase in $\mathrm{FEV}_{1}$ of over $10 \%$, but $6 \%$ had a fall of more than $10 \%$ (so, by inference, $64 \%$ had a change in $\mathrm{FEV}_{1}$ of less than $10 \%)$.

Subsequent studies have shown no significant benefit of short term use during exacerbations, ${ }^{6}$ improvements in spirometric indices but not in antibiotic use in more severely ill patients, ${ }^{7}$ a wide scatter of spirometric responses with a reduction in historical antibiotic use in children ${ }^{8}$ and, surprisingly, potentially harmful effects in a large group of patients with bronchiectasis not due to cystic fibrosis. ${ }^{9}$ Common to all these studies is the wide variability of responses in individual patients - the biological basis of which is unknown - and the failure to identify any predictors of individual responses.

Longer term data are now emerging and in this issue of Thorax Milla and colleagues ${ }^{10}$ report their results of a study on the effects of the introduction of DNase on the long term progress (nutritional state, spirometric indices and hospital admissions) in a large group of patients in a North American cystic fibrosis centre. Overall the $\mathrm{FEV}_{1}$ and allometric index (a measure of nutritional state) declined faster in the two years after the introduction of DNase than in the previous two years, and there was no change in hospital admission rates after treatment. Subgroup analysis again failed to reveal predictors of a good response apart from malnutrition at the time of prescription. While the conflict between these results and those from previous controlled trials may relate partly to study design and partly to the healthier population studied, these findings argue against the indiscriminate use of this treatment in the clinical setting.

How can the available data be used to direct treatment to maximal benefit? The cost of treatment cannot be ignored in this case as, at $£ 7442$ per patient per year for a once daily dosage, it equates broadly to the per capita UK cost of caring for cystic fibrosis patients in a specialist centre ${ }^{11}$ - that is, prescription to all patients would nearly double the cost of UK cystic fibrosis care. Cost of treatment is understandably an emotive issue with patients, carers, and managers

alike. A key principle of NHS health care is that effective treatment should not be denied on grounds of cost. Effectiveness and cost are, however, continuous variables and it is not difficult to construct a scenario where the indiscriminate use of an expensive intervention would yield overall significant benefit and yet be unjustifiable. To take an extreme example, if all patients with cystic fibrosis in the UK were to be admitted to hospital tomorrow for two weeks of intensive physiotherapy and intravenous antibiotics it is likely that the average $\mathrm{FEV}_{1}$ of cystic fibrosis patients in the UK would be improved, but only at the expense of much wasted effort, cost, and discomfort to the subset of fit individuals who did not benefit. An associated but more fundamental question is what level of benefit, and in what parameters, is significant? For DNase there is no consensus as to the appropriate weighting to attach to physiological measurements, antibiotic use, symptomatic scores, and nutritional parameters in assessing benefit. Penicillin in lobar pneumonia illustrates one end of a costbenefit spectrum, but at the other are treatments which use substantial health resources for benefits that are difficult to detect against the noise of clinical measurement variability in a variety of parameters.

The answer to these problems must lie in targeting treatment as accurately as possible to those individuals in whom benefit can be predicted or demonstrated beyond reasonable doubt. Unfortunately, the large trials of DNase do not permit "responders" to be identified prospectively using other clinical data. A possible solution lies in formalised individual trials of treatment ("n-of-1 trials" ${ }^{12}$ ) in which intensive serial measurements in an individual before and after the introduction of treatment are used to detect and quantify individual responses. In line with conventional design principles, it is important to incorporate randomisation, double blinding, and placebo control periods into such individual trials to retain objectivity. Such a protocol is now used to guide the use of DNase throughout Scotland ${ }^{13}$ and has been welcomed by patients, carers, and purchasers alike as an objective approach to targeting treatment.

High cost does not mean that use of a potentially effective treatment should be restricted, but it does put a firm obligation on carers to do everything possible to target the treatment objectively, to maximise measured individual benefit, and to follow closely the treated patients to ensure that these benefits are sustained.

J ALASTAIR INNES

Scottish Adult Cystic Fibrosis Service,

Western General Hospital,

Edinburgh EH4 2XU, UK

1 Shak S, Capon DJ, Hellmiss R, et al. Recombinant human DNase I reduces the viscosity of cystic fibrosis sputum. Proc Natl Acad Sci USA 1990;87:9188-92.

2 Ramsey BW, Astley SJ, Aitken ML, et al. Efficacy and safety of short-term administration of aerosolized recombinant human deoxyribonuclease in patients with cystic fibrosis. Am Rev Respir Dis 1993;148:145-151. 
3 Ranasinha C, Assoufi B, Christiansen D, et al. Efficacy and safety of shortterm administration of aerosolised recombinant human DNase I in adults with stable stage cystic fibrosis. Lancet 1993;342:199-202.

4 Costello CM, O'Connor CM, Finlay GA, et al. Effect of nebulised recombinant DNase on neutrophil elastase load in cystic fibrosis. Thorax 1996;51:619-23.

5 Fuchs HJ, Borowitz DS, Christiansen DH, et al. Effect of aerosolized recombinant human DNase on exacerbations of respiratory symptoms and pulmonary function in patients with cystic fibrosis. $N$ Engl $f$ Med 1994;331:637-42.

6 Wilmott RW, Amin RS, Colin AA, et al. Aerosolized recombinant human DNase in hospitalized cystic fibrosis patients with acute pulmonary exacerbations. Am f Respir Crit Care Med 1996;153:1914-7.

7 McCoy K, Hamilton S, Johnson C. Effects of 12-week administration of dornase alfa in patients with advanced cystic fibrosis lung disease. Chest 1996;110:889-95.
8 Davies J, Trindade M-T, Wallis C, et al. Retrospective review of the effects of DNase in children with cystic fibrosis. Pediatr Pulmonol 1997;23:243-8.

9 O'Donnell AE, Barker AF, Ilowite JS, et al. Treatment of idiopathic bronchiectasis with aerosolized recombinant human DNase 1. Chest 1998; 113:1329-34.

10 Milla CE. Long term effects of aerosolised rhDNase on pulmonary disease progression in patients with cystic fibrosis. Thorax 1998;53:0000-0.

11 Robson $\mathrm{M}$, Abbott J, Webb K, et al. A cost description of an adult CF unit and cost analyses of different categories of patients. Thorax 1992;47:6849 .

12 Guyatt G, Sackett D, Taylor W, et al. Determining optimal therapy: randomized trials in individual patients. N Engl F Med 1986;314:889-92.

13 Boellert FGE, Paton JY, Marshall TG, et al. Recombinant DNase in cystic fibrosis: a protocol for targeted introduction through n-of-1 trials. Eur Respir f 1998 (in press). 